\title{
FIP200 is Involved in Murine Pseudomonas Infection by Regulating HMGB1 Intracellular Translocation
}

\author{
Yi Li $i^{a, b, c}$ Chang-pei Gan ${ }^{a, b, c}$ Shuang Zhang ${ }^{a, b} \quad$ Xi-kun Zhou ${ }^{a, b} \quad$ Xue-feng Lia,b \\ Yu-quan Weia Jin-liang Yang ${ }^{\mathrm{a}}$ Min Wu ${ }^{\mathrm{b}}$ \\ aState Key Laboratory of Biotherapy, West China Hospital, West China Medical School, Sichuan \\ University, Chengdu, China, bepartment of Basic Sciences, University of North Dakota, Grand Forks, \\ ND, USA; 'The first two authors contributed equally to this work
}

\section{Key Words}

FIP200 • HMGB1 acetylation • Acute lung injury • Pulmonary infection

\begin{abstract}
Background: FIP200, a critical autophagy initiating protein, can participate in numerous cellular functions including cancer development; however, its functional role in $P$. aeruginos $a$ infection of alveolar macrophages is unknown. Methods: To investigate the role of FIP200 in host defense, we transfected murine alveolar macrophage MH-S cells with FIP200 siRNA. Having confirmed that FIP200 knockdown inhibited PAO1-induced autophagosme formation, we sought to characterize the underlying signaling pathways by immunoblotting. Further, we used fip200 KO mice to study the effects of fip200 deficiency on HMGB1 translocation. Results: We showed that Pseudomonas PAO1 strain infection facilitated autophagosome formation, whereas knockdown of FIP200 inhibited autophagosome formation and HMGB1 expression in MH-S cells. Silencing FIP200 impaired the translocation of HMGB1 to cytosol of MH-S cells and almost abolished acetylation of HMGB1 during PAO1 infection. In contrast, FIP200 overexpression facilitated the cytosol translocation of HMGB1 from nuclei and increased acetylation of HMGB1 in PAO1-infected MH-S cells. Importantly, expression and acetylation of HMGB1 were also significantly down-regulated in fip200 KO mice following PAO1 infection. Conclusions: Collectively, these findings elucidate that FIP200 may regulate expression and translocation of HMGB1 during PAO1 infection, which may indicate novel therapeutic targets to control pulmonary infection.
\end{abstract}




\section{Introduction}

Pseudomonas aeruginosa is commonly isolated from patients with hospital-acquired infection, and causes serious consequence in cystic fibrosis (CF) [1]. Treatments of this infection are usually difficult due to the impairment of multiple components in host immunity and fast development of antibiotic resistance, but the pathogenesis mechanism with this pathogen is incompletely understood $[2,3]$. Since alveolar macrophages (AM) are the sentinel of the initiation and integration of immune responses to microbial infection, we aimed to understand the molecular pathogenesis involved in AM defense against $P$. aeruginosa [4].

High mobility group box 1 (HMGB1) is a highly conserved, ubiquitous protein that is expressed in nearly all cell types. Not only can HMGB1 bind to double-stranded DNA and interact with other DNA-binding proteins to facilitate chromatin binding, but also function as a nuclear factor to enhance transcription in response to infection, inflammation, and tissue injury $[5,6]$. HMGB1 is a potential therapeutic target of local and systemic inflammatory diseases including acute lung injury, epithelial barrier dysfunction, and arthritis [7]. Extracellular HMGB1 released from inflammatory cells or necrotic cells can stimulate macrophages to secrete cytokines that can further amplify inflammatory responses [8]. In inflammatory diseases, such as sepsis, HMGB1 is translocated from the nucleus to the cytoplasm and actively secreted into the extracellular environment, where it interacts with several surface molecules, including Receptor for Advanced Glycation End-products (RAGE) and Toll-like Receptor 4 (TLR4) [9, 10]. Increased HMGB1 expression has been linked to infection progression by interfering with several signaling pathways, especially the autophagy pathway [11]. Huang et al. have reported that HMGB1-mediated autophagy contributed to chemotherapy in osteosarcoma by controlling the formation of Beclin1-Phosphatidylinositol 3-kinase Class 3 (PI3K3) complex [12]. As an upstream signal, Focal adhesion kinase family interacting protein of $200 \mathrm{kDa}$ (FIP200) is required for the interaction between HMGB1 and Beclin1, which then promotes Beclin1-PI3KC3 complex formation during autophagy [13].

Autophagy is essential for various cellular processes and associated with many human diseases, such as colon cancer, hepatitis B virus-associated hepatocellular carcinoma, diabetes, pulmonary infection, etc. [14-18]. FIP200, also known as RB1CC1 or RB1-inducible coiled-coil, is a component of the ULK1-Atg13-FIP200 complex, which is an essential autophagy initiator in mammalian cells [19]. Previous studies demonstrate that FIP200 is required for autophagy flux induced by infection in macrophages [20].

The role of FIP200 in the activation of macrophages during $P$. aeruginosa pulmonary infection remains unclear. Identifying effects of FIP200 on the production of HMGB1 by macrophages may help understand the molecular pathogenesis of P. aeruginosa infection. This study is designed to analyze the effects of FIP200 on HMGB1 translocation in macrophages during $P$. aeruginosa infection.

\section{Materials and Methods}

\section{Reagents}

P. aeruginosa strain PA01 wild-type (WT) was a gift from Stephen Lory (Harvard Medical School, Boston, MA). GFP-PA01 strain was obtained from Gerald Pier (Channing Laboratory, Harvard Medical School) [21]. The myc-FIP200 plasmid was a gift from DoHyung Kim (University of Minnesota, Minneapolis). The tandem RFP-GFP-LC3 plasmid was created and kindly provided by Tamotsu Yoshimori of Osaka University, Japan [22].

\section{Cell culture}

MH-S, a mouse macrophage-like cell line, was obtained from American Type Culture Collection (ATCC, Rockville, MD) and maintained following the supplier's instructions [23]. These cells were grown in 1640 containing 10\% FBS, 100 units $/ \mathrm{ml}$ penicillin, and $100 \mu \mathrm{g} / \mathrm{ml}$ streptomycin in a $95 \%$ air, 5\% $\mathrm{CO}_{2}$ humidified atmosphere at $37^{\circ} \mathrm{C}[24]$. 
Establishment of a murine model with $P$. aeruginosa infection

Cre-mediated-FIP200 conditional knockout (KO) mice were obtained from Dr. Junlin Guan at University of Michigan Medical School as described previously [25-27]. The conditional KO mice were generated by Cre-loxP approach, which mediated FIP200 deletion in endothelial cells [25-27]. Mice genotyping for FIP200 were performed by polymerase chain reaction analysis of tail DNA, essentially as described previously [26]. Six to
Table 1. Sequences of gene-specific primers used for PCR amplification

\begin{tabular}{ll}
\hline mRNA & Primer sequence \\
\hline GAPDH & R 5'-TGCCTGCTTCACCACCTTCT-3' \\
& F 5- AGGCCGGTGCTGAGTATGTC-3 \\
HMGB1 & R 5'- CTGCTTGT-CATCTGCAGCAG -3' \\
& F 5'- GGAGGAGCATAAGAAGAAGC -3' \\
RAGE & R 5'- TGTGTGGCCAC-CCATTCCAG -3' \\
& F 5'- GCCCTCCAGTACTACTCTCG -3' \\
FIP200 & R5'- CGATCTGCAGCCATGCATTC-3' \\
& F5'- GCCAATATCAAGCAAGCGCA-3' \\
\hline
\end{tabular}

eight week-old wild-type (WT) mice (C57BL/6J) were obtained from The Jackson Laboratory (Bar Harbor, ME) [28]. Mice were housed and bred in the animal facility at the University of North Dakota, and the animal experiments were performed in accordance with the institutional animal care and use committee guidelines (IACUC, approval number 1204-4). Bacteria were grown in Luria-Bertani (LB) broth overnight at $37^{\circ} \mathrm{C}$ with shaking. The bacteria were pelleted by centrifugation at $8000 \mathrm{~g}$ next day. To make sure that the bacteria grow until the mid-logarithmic phase, they were resuspended in $10 \mathrm{ml}$ of fresh LB broth [29]. We anesthetized mice with $45 \mathrm{mg} / \mathrm{kg}$ ketamine [30] and intranasally instilled to $1 \times 10^{7}$ (PAO1) colony-forming units (CFU) of $P$. aeruginosa, and mice were killed at $48 \mathrm{~h}$ following infection [31]. After bronchoalveolar lavage (BAL), the trachea and lung were excised for homogenization or fixed in $10 \%$ formalin.

\section{Infection experiments}

Bacteria were grown in LB broth overnight at $37{ }^{\circ} \mathrm{C}$ with shaking and pelleted by centrifugation at $8000 \mathrm{~g}$ next day. The optical density (OD) at $600 \mathrm{~nm}$ was measured, and the density was adjusted to 0.25 OD $\left(0.1 \mathrm{OD}=1 \times 10^{8}\right.$ cells $\left./ \mathrm{ml}\right)$. MH-S cells were washed once by PBS after overnight culture in serum-containing medium and changed to serum-free and antibiotic-free medium immediately before infection. Then cells were infected by PAO1 at an MOI (Multiplicity of Infection) of 10:1 for $3 \mathrm{~h}$. The CFU assay was performed after treating the infected cells with $100 \mu \mathrm{g} / \mathrm{ml}$ of polymyxin B [31, 32].

\section{RNA isolation and RT-PCR}

Total RNA was isolated from lung tissue using TRIzol reagent (Invitrogen, Carlsbad, CA) following the manufacturer's instructions. The concentration of RNA was determined and cDNA was generated using total RNA with the Reverse Transcriptase kit (Invitrogen). For amplification of the desired cDNA, specific primers were listed in Table 1. RT-PCR products were visualized on $1 \%$ agarose gels containing ethidium bromide following electrophoresis [33, 34].

\section{Cell transfection and confocal microscopy}

MH-S cells were respectively transfected with RFP-LC3 and RFP-GFP-LC3 plasmids, using LipofectAmine 2000 reagent (Invitrogen) in serum-free RPMI 1640 medium (Thermofisher Scientific, San Jose, CA) following the manufacturer's instructions. Transfected MH-S cells were also starved ( $6 \mathrm{~h})$ or treated with the autophagy inhibitor 3-MA ( $3 \mathrm{mM}, 3 \mathrm{~h})$ as a positive and negative control, respectively. For immunostaining, MH-S cells were grown in glass-bottomed dishes (MatTek, Ashland, MA) and fixed in 3.7\% paraformaldehyde, permeabilized with $0.2 \%$ Triton X-100 in PBS and incubated with blocking buffer for 30 min [35]. Then the dishes were incubated with an HMGB1 antibody (Santa Cruz Biotechnology, Santa Cruz, CA) at 1/500 dilution in blocking buffer overnight and washed three times with wash buffer. Images were captured after incubation with an appropriate secondary antibody containing FITC using an LSM 510 Meta confocal microscope (Carl Zeiss MicroImaging, Thornwood, NY), and processed using the software provided by the manufacturer [22]. The confocal microscopy images were then used to semi-quantitatively measure the percentage of cells with significant LC3 punctation staining (100 cells/sample). The threshold for positive expression was set to 10 visible LC3 puncta.

MTT assay

MH-S cells were seeded at a density of $5 \times 10^{3}$ per well in 96-well plates (Costar Corning, Rochester, NY). After incubation overnight, cells were transfected with FIP200 siRNA and control siRNA (Santa Cruz 
Biotechnology), respectively. Then cells were infected by PAO1 at an MOI of 10:1 for 3 h. 5 wells were included in each group. After $4 \mathrm{~h}$ incubation with 3-(4,5-dimethylthiazol-2-yl)-2,5-dimethyltetrazolium bromide dye at a final concentration of $5 \mu \mathrm{g} / \mathrm{ml}$ per well, the absorbance at $570 \mathrm{~nm}$ was measured with SpectraMax M5 (Molecular Devices), using wells without cells as blanks.

\section{Isolation of nuclear extracts}

Nuclear extracts were isolated following the instruction of NE-PER Nuclear And Cytoplasmic Extraction Reagents Kit (Pierce-Thermofisher Scientific). Cells were washed by suspending the cell pellet with phosphate-buffered saline. We added the CER I buffer and CER II buffer to swell the cells on ice for $10 \mathrm{~min}$ and then vortexed for $10 \mathrm{~s}$. Subsequently, samples were centrifuged for $10 \mathrm{~s}$ and the supernatant fraction was discarded. Pellets were resuspended in NER buffer and incubated on ice for 40 min for highsalt extraction. Cellular debris was removed by centrifugation for $10 \mathrm{~min}$ at $16,000 \mathrm{~g}$ and the supernatant fraction (containing DNA-binding proteins) was stored at $-80{ }^{\circ} \mathrm{C}$ until use. Protein concentrations were determined by the BCA Protein Assay Kit (Bio-Rad, Hercules, CA) [36].

\section{Western blot}

The samples from cells or tissues were lysed and quantified. Twenty micrograms of protein from murine lung tissue or MH-S cells were mixed with an equal volume of $2 \times$ SDS sample buffer, boiled for 5 min and then separated by 10\% SDS-polyacrylamide gel electrophoresis (PAGE). After electrophoresis, proteins were transferred to nitrocellulose membranes (Santa Cruz Biotechnology). Membranes were incubated with primary antibodies against FIP200, LC3, Beclin1, RAGE, ULK1, Histone, TLR4 and GAPDH (Santa Cruz Biotechnology). The antibodies against Atg13 and HMGB1 were from Cell Signaling Technology (Danvers, MA). Western blotting rabbit polyclonal antibodies, mouse polyclonal antibodies and goat polyclonal antibodies were obtained from Santa Cruz Biotechnology. Signals were visualized using an enhanced chemiluminescence detection kit (Santa Cruz Biotechnology). The protein signal was quantified by scanning densitometry using Quantity one imaging analysis (Bio-Rad).

\section{Immunoblotting and immunoprecipitation}

To obtain whole-cell lysates, MH-S cells or murine lung homogenates were homogenized in lysis buffer containing phosphatase inhibitor (1:1000) and Protease inhibitors (1:50, Roche, Indianapolis, IN). The samples were centrifuged at $12,000 \mathrm{rpm}$ for $10 \mathrm{~min}$ at $4^{\circ} \mathrm{C}$. Protein concentrations were determined by the BCA Protein Assay Kit (Bio-Rad) and then stored at $-80^{\circ} \mathrm{C}$ for immunoblotting analysis [37].

Whole-cell lysates were mixed with anti-FIP200 antibody (Cell Signaling Technology, Beverly, MA) or anti-HMGB1 antibody (Santa Cruz Biotechnology), respectively, which were coupled to agarose beads (Invitrogen). Nuclear Extracts were mixed with anti-acetylation on epsilon-amine groups of lysine residues antibody (Cell Signaling Technology) or anti-HMGB1 antibody (Santa Cruz Biotechnology) coupled with agarose beads. Immunoprecipitates were separated by SDS-PAGE and transferred to nitrocellulose transfer membranes (Santa Cruz Biotechnology). Signals were visualized using an ECL kit (Santa Cruz Biotechnology). The protein signal was quantified by scanning densitometry using Quantity one imaging analysis (Bio-Rad).

\section{Statistical analysis}

Statistical analysis was performed with the SPSS software system (SPSS for Windows, version 13.0; SPSS Inc., Chicago, IL). Parametric data were statistically analyzed by one-way ANOVA followed by post hoc tests. Differences in Non-parametric data were evaluated by the Mann-Whitney $U$ test. Data were expressed as means \pm SD. A significant difference was defined as $p<0.05$ [38].

\section{Results}

FIP200 knockdown inhibited PAO1-induced autophagosome formation and impaired phagocytosis function of MH-S cells

To investigate the role of FIP200 in alveolar macrophage defense against $P$. aeruginosa infection, we transfected murine MH-S cells with an RFP-LC3 plasmid and observed that 


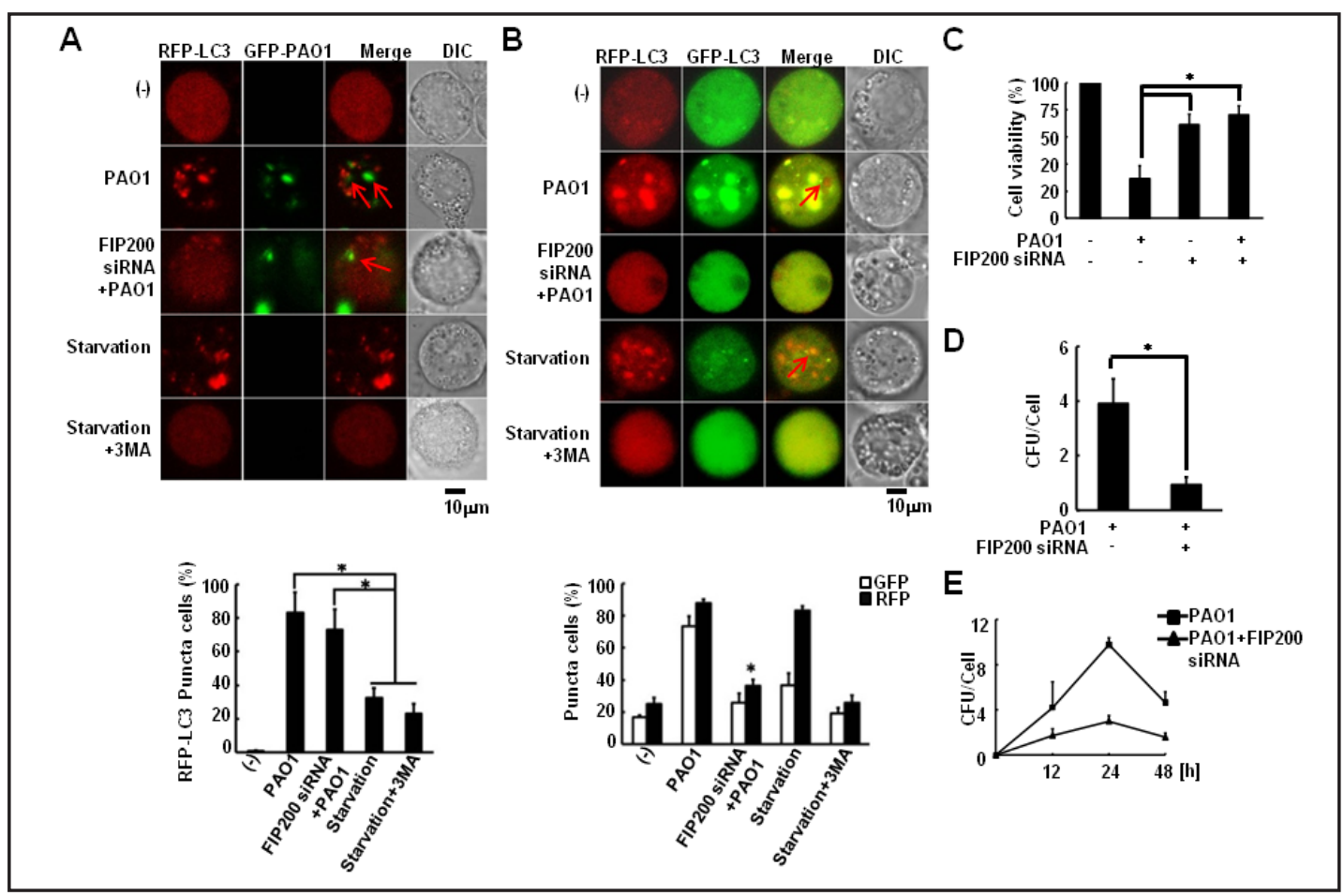

Fig. 1. FIP200 knockdown inhibited PAO1-induced autophagosome formation and impaired phagocytosis function of MH-S cells. A. MH-S cells were transfected with an RFP-LC3 plasmid and then infected for $3 \mathrm{~h}$ with GFP-PAO1 at an MOI of 10:1 induced significant LC3 punctation in MH-S cells. Transfected MH-S cells were also serum starved $(6 \mathrm{~h})$ or treated with the autophagy inhibitor 3-MA ( $3 \mathrm{mM}, 3 \mathrm{~h})$ as a positive and negative control, respectively. Before infection, the cells were transfected with FIP200 siRNA. B. MH-S cells were transfected with an RFP-GFP-LC3 plasmid and infected with PAO1 at an MOI of 10:1. Transfected MH-S cells were also serum starved $(6 \mathrm{~h})$ or treated with the autophagy inhibitor 3-MA ( $3 \mathrm{mM}, 3 \mathrm{~h})$. Before infection, the cells were transfected with FIP200 siRNA. C. FIP200 silencing improved MH-S cell survival upon PAO1 infection. Cells were infected by PAO1 at an MOI of 10:1 for $3 \mathrm{~h}$ and MTT assay was performed to determine host cell (MH-S) viability. Before infection, the cells were transfected with FIP200 siRNA. D. MH-S cells were infected by PAO1 at an MOI of 10:1 for $3 \mathrm{~h}$ and phagocytosis assay was performed. Before infection, the cells were transfected with FIP200 siRNA. E. MH-S cells were tested by phagocytosis assay in a time-dependent manner $(0,12,24$ and $48 \mathrm{~h})$ upon PAO1 infection at an MOI of 10:1. Before infection, the cells were transfected with FIP200 siRNA. The data represent means \pm SD of 3 independent experiments (one-way ANOVA followed by post hoc tests). Statistically significant differences are indicated by $*<<0.05$.

PA01 infection with an MOI of 10:1 induced significant LC3 punctation (83.33 $\pm 12.05 \%)$. However, FIP200 knockdown by siRNA resulted in a substantial downregulation of autophagy following PA01 infection with a decrease $(32.33 \pm 6.11 \%)$ of RFP-LC3 puncta (Fig. 1A). We next utilized a tandem RFP-GFP-LC3 construct to validate the observation and found that FIP200 knockdown affected autophagosome formation. The RFP-GFP-LC3 plasmid was designed to differentiate two major autophagic vesicles, the autophagosome and the autolysosome, thus excluding the likelihood of simple lysosome degradation blockade. When an autophagosome fuses with a lysosome, the GFP moiety degrades from the tandem protein, but RFP-LC3 maintains the red puncta to track autolysosomes [22]. After successful transfection with the tandem construct, FIP200 siRNA transfection significantly reduced red puncta $(36.67 \pm 3.51 \%)$ in $\mathrm{MH}-\mathrm{S}$ cells compared to control siRNA transfection $(88.33 \pm 1.53 \%)$ (Fig. 1B). PAO1 infection significantly decreased MH-S cell survival; however, FIP200 silencing improved MH-S cell survival after PAO1 invasion (Fig. 1C). FIP200 siRNAtransfected MH-S cells significantly decreased CFU after PAO1 infection (Fig. 1D). Finally, 
A

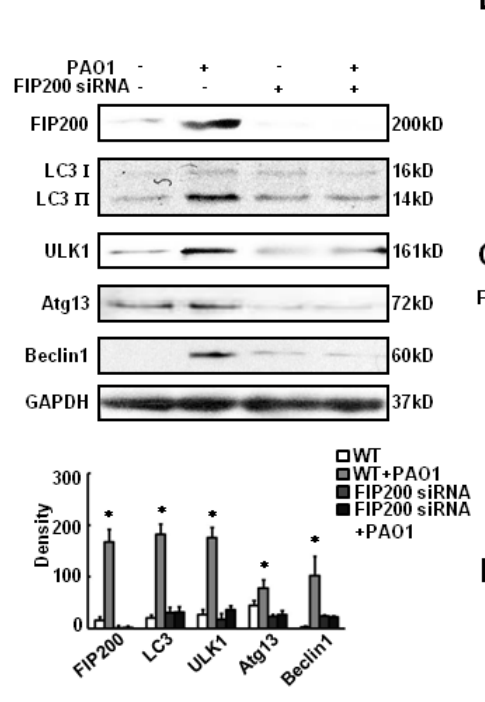

B
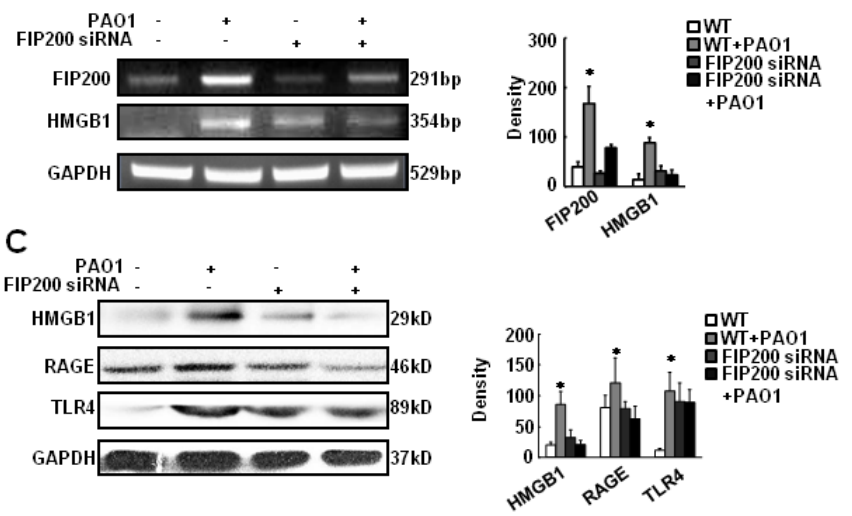

D

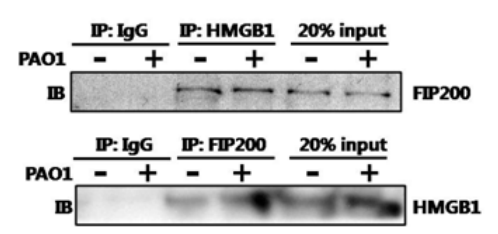

Fig. 2. FIP200 knockdown decreased the expression of HMGB1 during PAO1 infection. MH-S cells were transfected with FIP200 siRNA or control siRNA and then infected with PAO1 at an MOI of 10:1 for $3 \mathrm{~h}$. A. FIP200 knockdown decreased the expressions of LC3, Atg13, ULK1 and Beclin1 during PA01 infection in MH-S cells. B. FIP200 silencing in MH-S cells significantly reduced mRNA levels of FIP200 and HMGB1 after PA01 infection. C. FIP200 siRNA-transfection of MH-S cells significantly decreased expression in HMGB1, RAGE and TLR4 by western blotting. D. An interaction between FIP200 and HMGB1 identified by reciprocal coimmunoprecipitation. For western blotting we used HMGB1 antibody from Cell Signaling Technology (Danvers, MA). For immunoprecipitation we used anti-HMGB1 antibody from Santa Cruz Biotechnology. The data represent means \pm SD of 3 independent experiments (one-way ANOVA followed by post hoc tests). Statistically significant differences are indicated by ${ }^{*} p<0.05$.

we observed that FIP200 knockdown significantly decreased CFU upon infection in a timedependent manner (Fig. 1E). Overall, our results suggest that FIP200 may be required for PA01-induced autophagosome formation and phagocytosis function in macrophages.

FIP200 knockdown decreased the expression of HMGB1 during PAO1 infection

Having revealed that FIP200 knockdown inhibited PAO1-induced autophagosme formation, we sought to characterize the underlying signaling pathways. We examined the involvement of several canonical autophagic proteins related to FIP200, such as LC3, Atg13, ULK1 and Beclin1. PA01 infection up-regulated the expression of LC3, Atg13, ULK1 and Beclin1; however, FIP200 knockdown decreased the expressions of these proteins (Fig. 2A). We further analyzed the impact of FIP200 knockdown on HMGB1 expression. As shown in Fig. 2B, FIP200 silencing in MH-S cells significantly reduced mRNA level of HMGB1 after PAO1 infection (Fig. 2B). We also observed that FIP200 siRNA silencing in MH-S cells significantly decreased protein expression of HMGB1, RAGE and TLR4 following infection by western blotting (Fig. 2C). Thus we hypothesized that FIP200 may influence HMGB1 through molecular interaction. As expected, an interaction between FIP200 and HMGB1 occurred as identified by reciprocal coimmunoprecipitation (Co-IP) (Fig. 2D).

FIP200 knockdown impaired HMGB1 translocation during infection

We next examined the functional role of FIP200 on HMGB1 translocation and also found that FIP200 knockdown in MH-S cells inhibited HMGB1 translocation from nuclei 


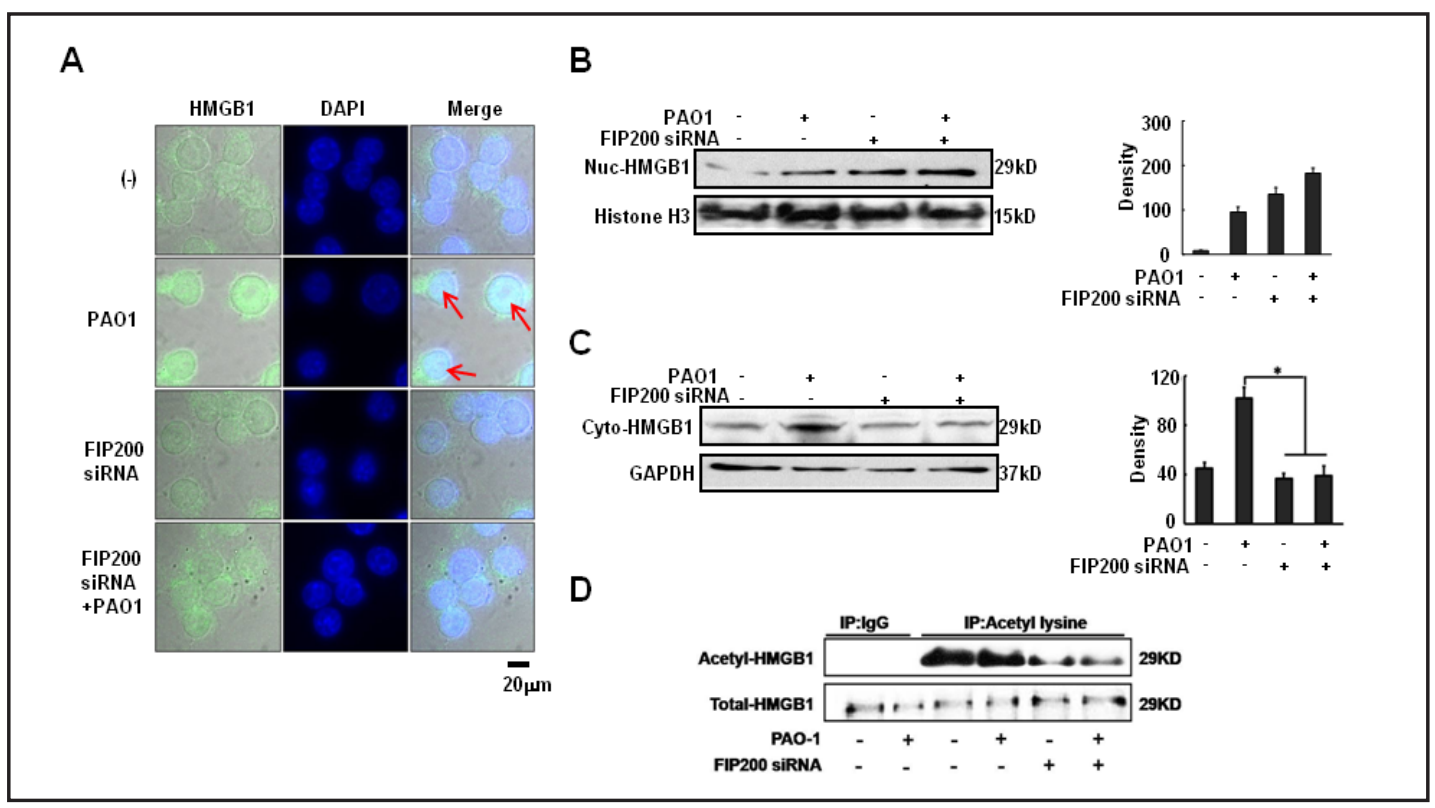

Fig. 3. FIP200 knockdown impaired HMGB1 cytosolic translocation from nuclei upon infection. MH-S cells were transfected with FIP200 siRNA or control siRNA and then infected with PAO1 at an MOI of 10:1 for 3 h. A. FIP200 knockdown in MH-S cells inhibited HMGB1 translocation to cytosol upon PAO1 infection. B. Nuclear HMGB1 was increased in PA01-infected MH-S cells upon FIP200 siRNA transfection. C. Cytosolic HMGB1 was decreased by FIP200 siRNA transfection post PAO1 infection. D. FIP200 siRNA transfection inhibited the acetylation of HMGB1. For western blotting we used an HMGB1 antibody from Cell Signaling Technology. For immunoprecipitation we used anti-HMGB1 antibody from Santa Cruz Biotechnology. The data represent means \pm SD of 3 independent experiments (one-way ANOVA followed by post hoc tests). Statistically significant differences are indicated by $* p<0.05$.

to cytoplasm upon PAO1 infection using immunocytochemistry staining (Fig. 3A). To mechanistically elucidate translocation of HMGB1 during PAO1 infection, we fractioned nuclear compartment from cytoplasm using a commercial kit (NE-PER Nuclear And Cytoplasmic Extraction Reagents). We observed that nuclear HMGB1 was increased by $86.67 \pm 12.42 \%$ in PA01-infected MH-S cells after FIP200 siRNA transfection, whereas cytosolic HMGB1 was decreased by $63.00 \pm 10.44 \%$ following the same treatment (Fig. 3B and C). Importantly, FIP200 silencing impaired the acetylation of the epsilon-amine groups on lysine residues in HMGB1 (Fig. 3D). These data indicate that FIP200 may regulate HMGB1 translocation by molecular interaction.

Overexpressing FIP200 facilitated cytosol translocation of HMGB1 during infection

To elucidate the functional role of FIP200 on HMGB1 translocation, we transfected MH-S cells with a myc-FIP200 plasmid and observed that FIP200 overexpression facilitated the cytosol translocation of HMGB1 from nuclei upon PA01 infection (Fig. 4A). Further, we detected that nuclear HMGB1 was decreased to $71.02 \pm 25.14 \%$ in PA01-infected MH-S cells after myc-FIP200 plasmid transfection, whereas cytosolic HMGB1 was increased to $250.11 \pm 24.95 \%$ (Fig. $4 \mathrm{~B}$ and C). Importantly, FIP200 overexpression increased the acetylation of the epsilon-amine groups on lysine residues in HMGB1 (Fig. 4D). These data indicate that FIP200 may positively regulate HMGB1 translocation by molecular interaction and acetylation of particular amino acids.

\section{fip200 deficiency was responsible for HMGB1 translocation in vivo}

To determine whether the response of HMGB1 pathway can occur in vivo, we used fip200 KO mice to study the effects of fip200 deficiency on HMGB1 translocation. As shown 


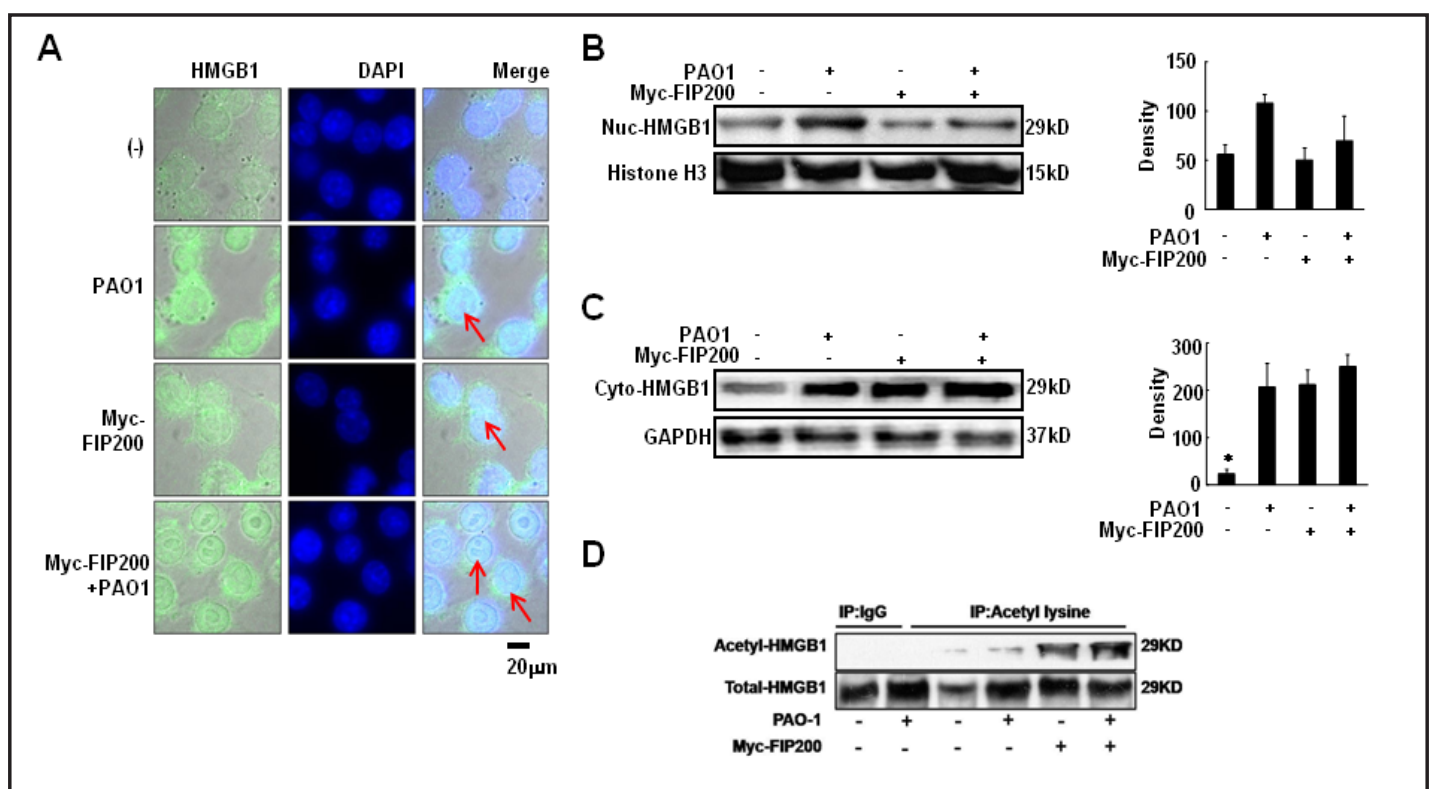

Fig. 4. FIP200 overexpression facilitated HMGB1 translocation under infection. MH-S cells were transfected with a myc-FIP200 plasmid and then infected with PAO1 at an MOI of 10:1 for 3 h. A. myc-FIP200 plasmid transfection in MH-S cells facilitated HMGB1 translocation after PAO1 infection. B. Nuclear HMGB1 was decreased in MH-S cells upon myc-FIP200 plasmid transfection and by PAO1 infection. C. Cytosolic HMGB1 in MH-S cells was increased by myc-FIP200 plasmid transfection and by PAO1 infection. D. myc-FIP200 plasmid transfection induced the acetylation of HMGB1. For western blotting we used an HMGB1 antibody from Cell Signaling Technology. For immunoprecipitation we used an anti-HMGB1 antibody from Santa Cruz Biotechnology. The data represent means \pm SD of 3 independent experiments (one-way ANOVA followed by post hoc tests). Statistically significant differences are indicated by $* p<0.05$.

in Fig. 5A, fip200 deficiency significantly reduced the expressions of HMGB1, RAGE and TLR4 in lung tissue after PAO1 infection. In addition, fip200 deficiency significantly decreased mRNA level of HMGB1 upon PAO1 infection (Fig. 5B). Furthermore, cytosolic HMGB1 was decreased by $70.10 \pm 10.21 \%$, whereas nuclear HMGB1 was increased by $150.11 \pm 25.03 \%$ in PA01-infected fip200 deficient mice (Fig. 5C and D). These data completely recapitulated the observation from cell models (Fig. 3 and 4). Furthermore, fip200 deficiency impaired the acetylation of the epsilon-amine groups of lysine residues in HMGB1 (Fig. 5E), which is also consistent with the data obtained from cell culture (Fig. 3 and 4). Altogether, our data strongly suggest that FIP200 plays a similar function in Pseudomonal infection in mice as seen in cultured cells by promoting HMGB1 activation and cytosolic translocation, and thus depleting FIP200 inhibits HMGB1 cytosolic translocation.

\section{Discussion}

In the current study, we demonstrated that FIP200 regulated HMGB1 translocation during $P$. aeruginosa infection. HMGB1 localized in the nucleus in resting cells, whereas PA01 infection decreased its nuclear levels but increased cytosolic levels. Consistent with this result, HMGB1 is previously reported to be released by exciting innate immunity with exogenous pathogen-derived molecules [39]. Once released, HMGB1 can bind to cellsurface receptors, such as RAGE and TLR4, and elicit various cellular responses including chemotactic cell penetration and proinflammatory cytokine production [40, 41]. Tang et al. reported that autophagy stimuli could induce HMGB1 cytosolic translocation from the nucleus, indicating that autophagy was required to enhance the cytosolic translocation of 


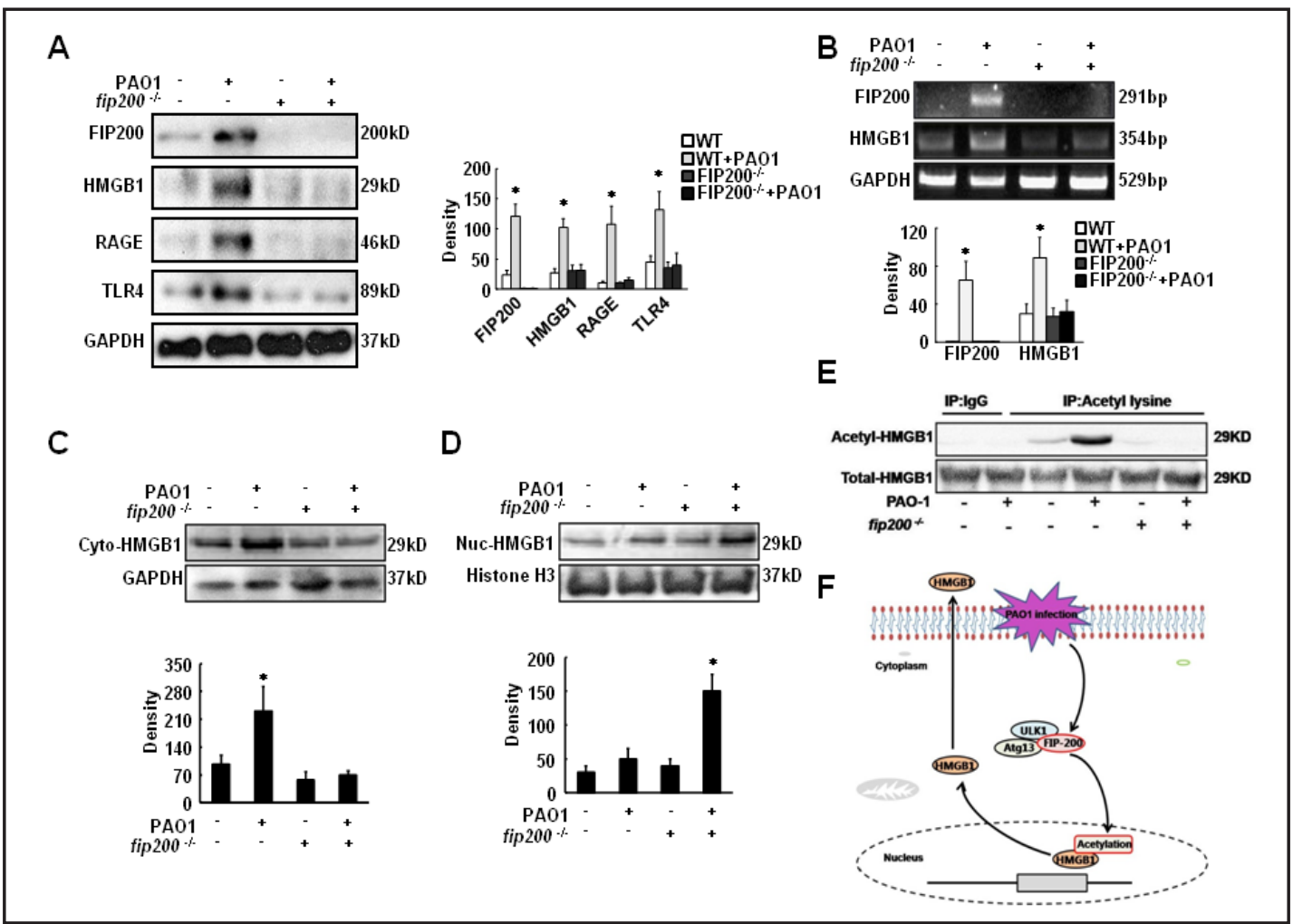

Fig. 5. fip200 deficiency impaired HMGB1 translocation in vivo. fip200 deficient mice and WT mice $(\mathrm{n}=5)$ were intranasally infected with $1 \times 10^{7} \mathrm{CFU} /$ mice PA01 for $48 \mathrm{~h}$. A. fip200 deficiency significantly reduced the expression of FIP200, HMGB1, RAGE and TLR4 in lung tissue following PAO1 infection. B. fip200 deficiency significantly reduced mRNA level of HMGB1 in lung tissue following PA01 infection. C. Cytosolic HMGB1 was decreased by fip200 deficiency post PAO1 infection. D. Nuclear HMGB1 was increased in PA01-infected MH-S cells upon fip200 deficiency. E. fip200 deficiency inhibited the acetylation of HMGB1. For western blotting we used an HMGB1 antibody from Cell Signaling Technology. For immunoprecipitation we used anti-HMGB1 antibody from Santa Cruz Biotechnology. The data represent means \pm SD of 3 independent experiments (one-way ANOVA followed by post hoc tests). Statistically significant differences are indicated by $* p<0.05$. F. Schematic illustration of FIP200-regulated HMGB1 translocation during P. aeruginosa infection.

HMGB1 [42]. Our findings showed that FIP200 silencing could suppress the expression of HMGB1, RAGE, K-Ras and TLR4 during PAO-1 infection. To further investigate the effect of FIP200 on the expression of HMGB1 in MH-S cells, we performed a Co-IP assay and found an interaction between FIP200 and HMGB1 following PAO1 infection. Thus, our data indicate that FIP200 may regulate HMGB1 translocation to cytosol following PA01 infection via direct molecular interaction with HMGB1.

Acetylation of HMGB1 induced by lipopolysaccharide and hydrogen peroxide triggers molecular translocation within the cell [43]. When HMGB1 is not acetylated, it stays in the nucleus; however, acetylation of lysine residues causes HMGB1 to translocate into the cytosol. Here, we showed that PAO1 infection induced acetylation of the epsilon-amine groups of lysine residues in HMGB1 and nuclear translocation of HMGB1. Importantly, FIP200 silencing significantly reduced the acetylation of HMGB1 and inhibited HMGB1 translocation in MH-S cells upon PAO1 infection. FIP200 knockdown increased the level of nuclear HMGB1 in PA01-infected MH-S cells, but decreased the level of cytosolic HMGB1. Furthermore, FIP200 overexpression facilitated cytosolic translocation of HMGB1 from nuclei and increased the acetylation of HMGB1 in PAO1 infected MH-S cells. To assess the physiological relevance, we examined the FIP200 functional role in bacterial infection in animals and obtained similar results in fip200 deficient mice. Our study also suggests that FIP200 functioning in response 
to pathogen recognition facilitates HMGB1 acetylation, leading to translocation of HMGB1. Post-translational modification of HMGB1 including acetylation at the site of lysines 2 and 11 in cells modifies the binding of HMGB1 to DNA and its extranuclear localization [44]. Further research may identify the exact acetylation sites of HMGB1 upon FIP200 regulation.

FIP200, which regulates diverse cellular functions through its interaction with multiple other proteins, is also required for autophagy flux [45, 46]. However, the role of FIP200 in $P$. aeruginosa infection remains undefined. Here, we demonstrated that FIP200 could affect extracellular bacterial clearance by facilitating autophagy flux, as FIP200 knockdown significantly decreased bacterial burdens of murine alveolar macrophage MH-S cells. Despite focusing on alveolar macrophages, alveolar epithelial cells could also serve as an inflammatory and immune responder and play important roles in tissue injury [47]. Thus, further studies can also address the mechanisms that are responsible for FIP200 in alveolar epithelial cells upon P. aeruginosa infection.

In contrast to the intense interest in autophagy's roles in cell viability, tumor, and inflammatory disease, little is known about how FIP200 regulates cellular functions in infectious disease. Our results showed that FIP200 siRNA transfection reduced the expression of HMGB1, RAGE and K-Ras in PAO1-infected MH-S cells. fip200 deficiency decreased levels of HMGB1, RAGE and K-Ras in PA01-infected mice. Mechanistically, we identified an interaction between FIP200 and HMGB1. Moreover, fip200 deficiency impaired acetylation of HMGB1. Collectively, this study reveals that FIP200 upon PA01 pulmonary infection down-regulates HMGB1 expression and its cytosolic translocation. Our findings suggest that FIP200 may play a regulatory role in alveolar macrophage-mediated host defense against $P$. aeruginosa infection, and serve as a target for therapy of Gram-negative bacterial infection.

\section{Acknowledgements}

We thank Sarah Rolling of the University of North Dakota imaging core for help with confocal imaging. This work was supported by Flight Attendant Medical Research Institute (FAMRI, Grant \#103007), NIH AI101973-01 and AI097532-01A1 to M.W.

\section{Disclosure Statement}

The authors declare no financial or commercial conflict of interest.

\section{References}

1 Lyczak JB, Cannon CL, Pier GB: Lung infections associated with cystic fibrosis. Clin Microbiol Rev 2002;15:194-222.

-2 Renna M, Schaffner C, Brown K, Shang S, Tamayo MH, Hegyi K, Grimsey NJ, Cusens D, Coulter S, Cooper J, Bowden AR, Newton SM, Kampmann B, Helm J, Jones A, Haworth CS, Basaraba RJ, DeGroote MA, Ordway DJ, Rubinsztein DC, Floto RA: Azithromycin blocks autophagy and may predispose cystic fibrosis patients to mycobacterial infection. J Clin Invest 2011;121:3554-3563.

-3 van de Weert-van Leeuwen PB, Arets HGM, van der Ent CK, Beekman JM: Infection, inflammation and exercise in cystic fibrosis. Resp Res 2013;14:32.

4 Hayden MS, Ghosh S: Nf-kb in immunobiology. Cell Res 2011;21:223-244.

5 Li J, Kokkola R, Tabibzadeh S, Yang R, Ochani M, Qiang X, Harris HE, Czura CJ, Wang H, Ulloa L, Wang H, Warren HS, Moldawer LL, Fink MP, Andersson U, Tracey KJ, Yang H: Structural basis for the proinflammatory cytokine activity of high mobility group box 1. Mol Med (Cambridge, Mass) 2003;9:3745.

6 Andersson U, Erlandsson-Harris H, Yang H, Tracey KJ: Hmgb1 as a DNA-binding cytokine. J Leukocyte Biol 2002;72:1084-1091. 
7 Yang H, Wang H, Czura CJ, Tracey KJ: The cytokine activity of hmgb1. J Leukocyte Biol 2005;78:1-8.

8 Lotze MT, Tracey KJ: High-mobility group box 1 protein (hmgb1): Nuclear weapon in the immune arsenal. Nat Rev Immunol 2005;5:331-342.

-9 Erlandsson Harris H, Andersson U: Mini-review: The nuclear protein hmgb1 as a proinflammatory mediator. Eur J Immunol 2004;34:1503-1512.

10 Akira S, Takeda K: Toll-like receptor signalling. Nat Rev Immunol 2004;4:499-511.

11 Zhang Y, Yang JW, Ren X, Yang J-M: Nac1 and hmgb1 enter a partnership for manipulating autophagy. Autophagy 2011;7:1557-1558.

12 Huang J, Ni J, Liu K, Yu Y, Xie M, Kang R, Vernon P, Cao L, Tang D: Hmgb1 promotes drug resistance in osteosarcoma. Cancer Res 2012;72:230-238.

13 Huang J, Liu K, Yu Y, Xie M, Kang R, Vernon PJ, Cao L, Tang D, Ni J: Targeting hmgb1-mediated autophagy as a novel therapeutic strategy for osteosarcoma. Autophagy 2012;8:275-277.

14 Lan S-H, Wu S-Y, Zuchini R, Lin X-Z, Su I-J, Tsai T-F, Lin Y-J, Wu C-T, Liu H-S: Autophagy suppresses tumorigenesis of hepatitis b virus-associated hepatocellular carcinoma through degradation of mir-224. Hepatology 2014;59:505-517.

15 Zhou X, Li C, Lu X: Autophagy activation by rapamycin improves left ventricular function in diabetic rats. Int J Cardiol 2013;168:4429-4431.

16 Verdoodt B, Vogt M, Schmitz I, Liffers S-T, Tannapfel A, Mirmohammadsadegh A: Salinomycin induces autophagy in colon and breast cancer cells with concomitant generation of reactive oxygen species. Plos One 2012;7:e44132.

-17 Abdulrahman BA, Khweek AA, Akhter A, Caution K, Kotrange S, Abdelaziz DHA, Newland C, RosalesReyes R, Kopp B, McCoy K, Montione R, Schlesinger LS, Gavrilin MA, Wewers MD, Valvano MA, Amer AO: Autophagy stimulation by rapamycin suppresses lung inflammation and infection by burkholderia cenocepacia in a model of cystic fibrosis. Autophagy 2011;7:1359-1370.

-18 Pierdominici M, Vomero M, Barbati C, Colasanti T, Maselli A, Vacirca D, Giovannetti A, Malorni W, Ortona E: Role of autophagy in immunity and autoimmunity, with a special focus on systemic lupus erythematosus. The FASEB J 2012;26:1400-1412.

19 Hosokawa N, Hara T, Kaizuka T, Kishi C, Takamura A, Miura Y, Iemura S-i, Natsume T, Takehana K, Yamada N, Guan J-L, Oshiro N, Mizushima N: Nutrient-dependent mtorc1 association with the ulk1-atg13-fip200 complex required for autophagy. Mol Biol Cell 2009;20:1981-1991.

20 He C, Klionsky DJ: Regulation mechanisms and signaling pathways of autophagy. Annu Rev Genet 2009;43:67.

21 Kannan S, Audet A, Huang H, Chen L-j, Wu M: Cholesterol-rich membrane rafts and lyn are involved in phagocytosis during pseudomonas aeruginosa infection. J Immunol 2008;180:2396-2408.

-22 Yuan K, Huang C, Fox J, Laturnus D, Carlson E, Zhang B, Yin Q Gao H, Wu M: Autophagy plays an essential role in the clearance of pseudomonas aeruginosa by alveolar macrophages. J Cell Sci 2012;125:507-515.

23 Matsunaga K, Klein TW, Friedman H, Yamamoto Y: Alveolar macrophage cell line mh-s is valuable as an in vitro model for legionella pneumophila infection. Am J Resp Cell Mol 2001;24:326-331.

-24 Wisniowski PE, Spech RW, Wu MIN, Doyle NA, Pasula R, Martin WJ: Vitronectin protects alveolar macrophages from silica toxicity. Am J Resp Crit Care 2000;162:733-739.

25 Shen T-L, Park AY-J, Alcaraz A, Peng X, Jang I, Koni P, Flavell RA, Gu H, Guan J-L: Conditional knockout of focal adhesion kinase in endothelial cells reveals its role in angiogenesis and vascular development in late embryogenesis. J Cell Biol 2005;169:941-952.

26 Gan B, Peng X, Nagy T, Alcaraz A, Gu H, Guan J-L: Role of fip200 in cardiac and liver development and its regulation of tnf $\alpha$ and tsc-mtor signaling pathways. J Cell Biol 2006;175:121-133.

27 Wei H, Gan B, Wu X, Guan J-L: Inactivation of fip200 leads to inflammatory skin disorder, but not tumorigenesis, in conditional knock-out mouse models. J Biol Chem 2009;284:6004-6013.

28 Zhang Y, Goldman S, Baerga R, Zhao Y, Komatsu M, Jin S: Adipose-specific deletion of autophagy-related gene 7 (atg7) in mice reveals a role in adipogenesis. P Natl Acad Sci USA 2009;106:19860-19865.

-29 Kannan S, Huang H, Seeger D, Audet A, Chen Y, Huang C, Gao H, Li S, Wu M: Alveolar epithelial type ii cells activate alveolar macrophages and mitigate P. aeruginosa infection. Plos One 2009;4:e4891.

Wu M, Hussain S, He Y-H, Pasula R, Smith PA, Martin WJ: Genetically engineered macrophages expressing ifn- $\gamma$ restore alveolar immune function in scid mice. P Natl Acad Sci USA 2001;98:14589-14594. 
-31 Yuan K, Huang C, Fox J, Gaid M, Weaver A, Li G, Singh BB, Gao H, Wu M: Elevated inflammatory response in caveolin-1-deficient mice with pseudomonas aeruginosa infection is mediated by stat3 protein and nuclear factor кb (nf-kb). J Biol Chem 2011;286:21814-21825.

32 Li X, Zhou X, Ye Y, Li Y, Li J, Privratsky B, Wu E, Gao H, Huang C, Wu M: Lyn regulates inflammatory responses in klebsiella pneumoniae infection via the p38/nf-кb pathway. Eur J Immunol 2013;44:763-773.

33 Guo Q Shen N, Yuan K, Li J, Wu H, Zeng Y, Fox J, Bansal AK, Singh BB, Gao H, Wu M: Caveolin-1 plays a critical role in host immunity against klebsiella pneumoniae by regulating stat5 and akt activity. Eur J Immunol 2012;42:1500-1511.

-34 Li G, Yuan K, Yan C, Fox III J, Gaid M, Breitwieser W, Bansal AK, Zeng H, Gao H, Wu M: 8-oxoguanine-DNA glycosylase 1 deficiency modifies allergic airway inflammation by regulating stat 6 and il-4 in cells and in mice. Free Radical Bio Med 2012;52:392-401.

-35 Zhang D, Wu M, Nelson DE, Pasula R, Martin WJ, II: Alpha-1-antitrypsin expression in the lung is increased by airway delivery of gene-transfected macrophages. Gene Ther 2003;10:2148-2152.

36 Kannan S, Pang H, Foster DC, Rao Z, Wu M: Human 8-oxoguanine DNA glycosylase increases resistance to hyperoxic cytotoxicity in lung epithelial cells and involvement with altered mapk activity. Cell Death Differ 2005;13:311-323.

37 He Y-H, Wu M, Kobune M, Xu Y, Kelley MR, Martin WJ: Expression of yeast apurinic/apyrimidinic endonuclease (apn1) protects lung epithelial cells from bleomycin toxicity. Am J Resp Cell Mol 2001;25:692-698.

38 Wu M, Pasula R, Smith PA, Martin WJ: Mapping alveolar binding sites in vivo using phage peptide libraries. Gene Ther 2003;10:1429-1436.

-39 Andersson U, Tracey KJ: Hmgb1 is a therapeutic target for sterile inflammation and infection. Annu Rev Immunol 2011;29:139-162.

-40 Riuzzi F, Sorci G, Donato R: The amphoterin (hmgb1)/receptor for advanced glycation end products (rage) pair modulates myoblast proliferation, apoptosis, adhesiveness, migration, and invasiveness: Functional inactivation of rage in 16 myoblasts results in tumor formation in vivo. J Biol Chem 2006;281:8242-8253.

41 Ibrahim ZA, Armour CL, Phipps S, Sukkar MB: Rage and tlrs: Relatives, friends or neighbours? Mol Immunol 2013;56:739-744.

42 Tang D, Kang R, Livesey KM, Cheh C-W, Farkas A, Loughran P, Hoppe G, Bianchi ME, Tracey KJ, Zeh HJ, Lotze MT: Endogenous hmgb1 regulates autophagy. J Cell Biol 2010;190:881-892.

43 Funayama A, Shishido T, Netsu S, Narumi T, Kadowaki S, Takahashi H, Miyamoto T, Watanabe T, Woo C-H, Abe J-i: Cardiac nuclear high mobility group box 1 prevents the development of cardiac hypertrophy and heart failure. Cardiovasc Res 2013;99:657-664.

44 Bonaldi T, Talamo F, Scaffidi P, Ferrera D, Porto A, Bachi A, Rubartelli A, Agresti A, Bianchi ME: Monocytic cells hyperacetylate chromatin protein hmgb1 to redirect it towards secretion. EMBO J 2003;22:55515560 .

45 Lee JY: The regulation of signaling in hematopoietic stem cell maintenance, The University of Michigan, 2010.

46 Kobayashi S, Yoneda-Kato N, Itahara N, Yoshida A, Kato J-y: The cop1 e3-ligase interacts with fip200, a key regulator of mammalian autophagy. BMC Biochem 2013;14:1.

47 Kannan S, Wu M: Respiratory stem cells and progenitors: Overview, derivation, differentiation, carcinogenesis, regeneration and therapeutic application. Curr Stem Cell Res Ther 2006;1:37-46. 


\section{Errata}

The original article by Li et al., entitled "FIP200 is involved in murine Pseudomonal infection by regulating HMGB1 intracellular translocation” [Cell Physiol Biochem 2014;33:1733-1744 (DOI: 10.1159/000362954)], contain several errors in literature citations, which are corrected as below.

Ref. 30 on Page 1343, P Natl Acad Sci USA 2001;98:14589-14594. P should be Proc

Ref. 32 on Page 1344, 2013 should be 2014

Ref. 36 on Page 1344, 2005 should be 2006

Ref. 37 on Page 1344, He Y-H, Martin WJ, should be He YH, Martin WJ $2^{\text {nd }}$. 